\title{
Spatially and genetically distinct control of seed germination by phytochromes $A$ and $B$
}

\author{
Keun Pyo Lee, ${ }^{1}$ Urszula Piskurewicz, ${ }^{1}$ Veronika Turečková, ${ }^{2}$ Solenne Carat, ${ }^{3}$ Richard Chappuis, ${ }^{1}$ \\ Miroslav Strnad, ${ }^{2}$ Christian Fankhauser, ${ }^{4}$ and Luis Lopez-Molina ${ }^{1,5}$ \\ ${ }^{1}$ Département de Biologie Végétale, 30, quai Ernest-Ansermet-Sciences III, Université de Genève, 1211 Genève 4, Switzerland; \\ ${ }^{2}$ Laboratory of Growth Regulators, Palacky University, Institute of Experimental Botany, Academy of Sciences of the Czech \\ Republic, 78371 Olomouc, Czech Republic; ${ }^{3}$ Bioinformatics and Biostatistics Core Facility, EPFL School of Life Sciences, 1015 \\ Lausanne, Switzerland; ${ }^{4}$ Center for Integrative Genomics, University of Lausanne, CH-1015 Lausanne, Switzerland
}

Phytochromes phyB and phyA mediate a remarkable developmental switch whereby, early upon seed imbibition, canopy light prevents phyB-dependent germination, whereas, later on, it stimulates phyA-dependent germination. Using a seed coat bedding assay where the growth of dissected embryos is monitored under the influence of dissected endosperm, allowing combinatorial use of mutant embryos and endosperm, we show that canopy light specifically inactivates phyB activity in the endosperm to override phyA-dependent signaling in the embryo. This interference involves abscisic acid (ABA) release from the endosperm and distinct spatial activities of phytochrome signaling components. Under the canopy, endospermic ABA opposes phyA signaling through the transcription factor (TF) ABI5, which shares with the TF PIF1 several target genes that negatively regulate germination in the embryo. ABI5 enhances the expression of phytochrome signaling genes PIF1, SOMNUS, GAI, and $R G A$, but also of $\mathrm{ABA}$ and gibberellic acid (GA) metabolic genes. Over time, weaker ABA-dependent responses eventually enable phyA-dependent germination, a distinct type of germination driven solely by embryonic growth.

[Keywords: ABI5; DELLA factors; abscisic acid; phyA; phyB; seed germination]

Supplemental material is available for this article.

Received April 14, 2012; revised version accepted July 9, 2012.

Colonization of land is a major chapter in the history of plant evolution. Essential for this success has been the appearance of seeds, a key structure allowing plant propagation (Linkies et al. 2010). In Arabidopsis, mature seeds consist of an outer layer of dead tissue, the testa, underneath which the endosperm single-cell layer surrounds the embryo. Seed germination chronologically involves testa rupture followed by concomitant endosperm rupture and radicle elongation, cotyledon expansion, and greening (Bentsink and Koornneef 2008; Weitbrecht et al. 2011). Multiple levels of regulation have appeared during evolution to control germination in response to environmental cues such as water potential or light quality (Finkelstein et al. 2008; Kami et al. 2010). In addition, newly produced seeds are dormant; i.e., they repress germination despite favorable germination conditions (Bentsink and Koornneef 2008).

Nondormant seed germination is tightly dependent on the relative intensity of red light $(\mathrm{R})$ and far-red light $(\mathrm{FR})$,

${ }^{5}$ Corresponding author

E-mail luis.lopezmolina@unige.ch

Article is online at http://www.genesdev.org/cgi/doi/10.1101/gad.194266.112. sensed in Arabidopsis by five phytochromes (phyA-phyE) (Rockwell et al. 2006; Kami et al. 2010; Strasser et al. 2010). phyB and phyA play a key role in regulating lightdependent germination responses (Shinomura et al. 1994, 1996). However, phyC, phyD, and phyE have also been reported to play a role in some conditions (Poppe and Schafer 1997; Hennig et al. 2002; Franklin et al. 2003; Dechaine et al. 2009; Strasser et al. 2010). Phytochromes are synthesized in a signaling-inactive state known as Pr. Upon $\mathrm{R}$ absorption, they photoconvert reversibly to a FRabsorbing and signaling-active form known as Pfr. Unlike phyB and the other phytochromes, phyA can initiate downstream events in response to very low fluences of a wide spectrum of light (very low fluence response [VLFR]) and high levels of FR (high irradiance response [HIR]) (Shinomura et al. 1996).

Early upon seed imbibition, seed germination depends on the activation of phyB by sunlight enriched in $\mathrm{R}$ (hereafter, germination promoted by the active Pfr form of phyB is referred to as phyB-dependent germination). The timing of the light irradiation can have a profound influence on germination responses. Indeed, remarkably and paradoxically, early upon seed imbibition, a FR pulse 
inhibits phyB-dependent germination (i.e., an inhibition involving FR-dependent conversion of phyB into its inactive Pr form), whereas a later, second FR pulse (e.g., $48 \mathrm{~h}$ after the first FR pulse) will stimulate phyA-dependent germination (i.e., a stimulation involving light-dependent conversion of phyA into its active Pfr form) (Reed et al. 1994; Shinomura et al. 1994). The mechanisms underlying these opposite germination responses were not thoroughly investigated but were proposed to result from low phyA protein levels relative to phyB protein levels early upon seed imbibition (Shinomura et al. 1996).

It is usually assumed that phytochromes control the relative levels of gibberellic acid (GA) and abscisic acid (ABA), two hormones promoting and repressing germination, respectively. Recent studies have shown that only ABA prevents embryonic growth, whereas GA negatively controls ABA levels, although GA also promotes testa rupture (Piskurewicz et al. 2008, 2009). This study therefore views phytochrome signaling as a pathway whose final output is to control ABA levels.

Early phytochrome signaling involves the basic helixloop-helix (bHLH) transcription factor (TF) PIF1 (also called PIL5), which is destabilized upon interaction with the Pfr form of phytochromes. When phytochromes are in the inactive Pr form, PIF1 is stabilized and represses germination. In an unknown manner, PIF1 represses the GA synthesis genes GA3ox1 and GA3ox2 (referred to as GA3ox1/2) and stimulates the expression of the ABA synthesis genes NCED6 and NCED9 (referred to as NCED6/9) (Yamaguchi et al. 1998; Oh et al. 2006). Furthermore, PIF1 stimulates the expression of SOMNUS (SOM), encoding a CCHHtype zinc finger protein. SOM also represses germination by repressing and stimulating GA and ABA synthesis gene expression, respectively, in an unknown manner (Kim et al. 2008; Park et al. 2011). Finally, PIF1 stimulates the expression of GAI and RGA, encoding DELLA factors (Oh et al. 2004, 2007; Piskurewicz et al. 2008, 2009). When GA levels are low, RGL2, GAI, and RGA repress germination by stimulating $\mathrm{ABA}$ levels in an unknown manner (Piskurewicz et al. 2008, 2009). In turn, ABA levels block embryonic growth by stimulating the expression of the ABA response TFs $A B I 3$ and $A B I 5$, which repress endosperm rupture and cotyledon greening (Lopez-Molina and Chua 2000; Lopez-Molina et al. 2002).

Recent work has shown that in dormant seeds, GA fails to promote the degradation of DELLA factors so that DELLAs constitutively stimulate ABA synthesis, thus blocking germination. In this context, RGL2 stimulates in the endosperm the production and release of $A B A$ toward the embryo to block its germination. This was shown using a seed coat bedding assay (SCBA) in which dissected embryos are cultured on a layer of dissected seed coats, allowing combinatorial use of different embryonic and seed coat materials (Lee et al. 2010).

Here, we show that the endosperm operates as a key tissue controlling germination in response to light cues. Using SCBAs, we show that the endosperm mediates FR repression of phyB-dependent germination, whereas FR stimulation of phyA-dependent germination occurs only in the embryo. These responses specifically involve the light signaling genes PIF1 and RGL2 in the endosperm and PIF1, SOM, GAI, and RGA in the embryo, where they regulate the expression of GA and ABA synthetic genes in each tissue. Early upon seed imbibition, FR inactivation of phyB leads to ABA synthesis and release from the endosperm to prevent phyA-dependent promotion of germination in the embryo. This involves an extended regulatory network where $\mathrm{ABA}$ overrides phyA signaling by interfering with the expression of light signaling genes and GA and ABA metabolic genes. We show that this is associated with the direct binding of ABI5 to promoter sequences in vivo. Over time, a weakening of ABAdependent responses takes place, thus allowing phyAdependent germination after a later light treatment. This results in a phyA-dependent "explosive" germination unlike phyB-dependent germination, where the embryo exits the seed coat without prior testa rupture, since it occurs in the presence of FR-dependent inactivation of phyB in the endosperm.

\section{Results}

Early phy A signaling in the embryo is opposed by the seed coat

Early seed germination steps involve testa rupture followed by endosperm rupture (Muller et al. 2006). We observed that phyA-dependent germination after a second FR pulse is "explosive" (i.e., involving endosperm rupture without prior visible testa rupture), unlike phyB-dependent germination (Fig. 1A). This suggested that phyA and phyB do not signal in the same tissues.

To address this hypothesis, we monitored the growth of light-irradiated embryos after dissecting their seed coats shortly upon seed imbibition. The dissection procedure did not significantly alter phyA or phyB protein levels (Supplemental Fig. 1A). Strikingly, FR-treated wild-type, phy $B$, or phy $B C D E$ coatless embryos were able to germinate, unlike FR-treated phy $A$ and phyACDE embryos (Fig. 1B; Supplemental Fig. 1B). This indicated that phyAdependent germination can occur early upon imbibition, provided that the seed coat is removed. We wished to verify that coatless embryos indeed display phyA-dependent changes in GA and ABA synthesis gene expression. Wildtype, phyB, or phyBCDE cultured embryos had high GA3ox1/2 and low NCED6/9 expression relative to wildtype, $p h y B$, or phyBCDE embryos dissected from seeds cultured in the same conditions (Fig. 1C; Supplemental Fig. 1C). In contrast, GA3ox1/2 and NCED6/9 expression in FR-treated phy $A$ and phy $A C D E$ cultured embryos was similar to that in embryos dissected from phy $A$ and phyACDE seeds cultured in the same conditions (Fig. 1C; Supplemental Fig. 1C). The dissection procedure was not associated with significant contamination of the embryo with seed coat tissue or vice versa (see the Material and Methods; Supplemental Fig. 1D).

Previous work showed that ABA acts downstream from GA to block germination and that GA negatively regulates ABA levels (Piskurewicz et al. 2009). We wished to verify that absence of coatless phyA and phyACDE 

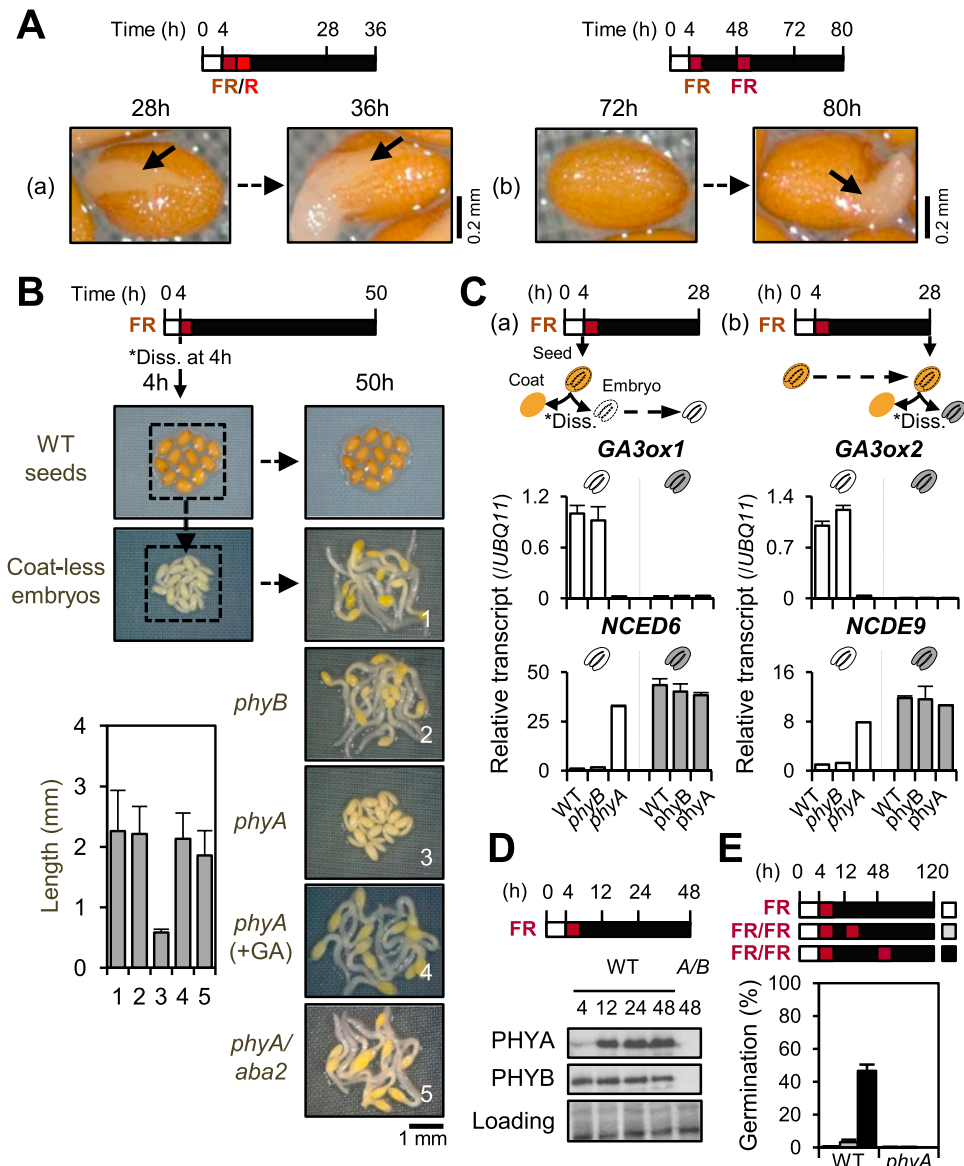

(h) 04 (b)

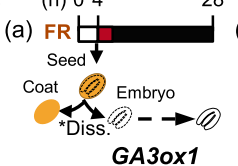

(i)

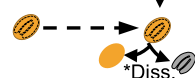

GA3OX2

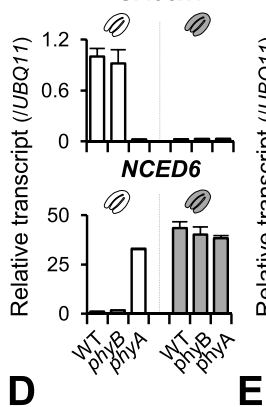

(h) $04 \begin{array}{lllll} & 12 & 24 & 48\end{array}$

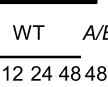

PHYA

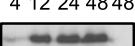

PHYB

Loading

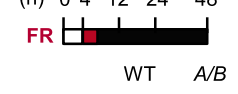

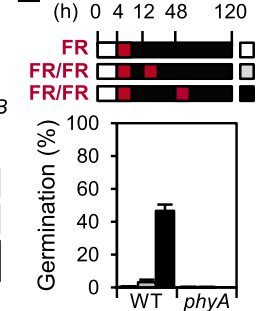

Figure 1. Early phyA-dependent germination of coatless embryos. phyA protein levels poorly correlate with phyA-dependent germination. $(A$, panel $a)$ phyB-dependent wild-type (WT) germination was induced after a FR pulse immediately followed by a $\mathrm{R}$ pulse $(F R / R)$. Pictures show seeds at the indicated times after seed imbibition. Arrows show testa rupture $(28 \mathrm{~h})$ and endosperm rupture $(36 \mathrm{~h})$ events. (Panel b) phyA-dependent wild-type germination was induced after two consecutive FR pulses (FR/ FR). The arrow $(80 \mathrm{~h})$ shows an endosperm rupture event without prior visible testa rupture events $(72$ h). (B) Diagram showing the procedure for dissection and culture of embryos: Seeds are imbibed $4 \mathrm{~h}$ under white light in normal germination medium. Next, seed coats were dissected (Diss.), and embryos were irradiated with FR before incubation in darkness. Pictures show wild-type seeds and coatless wild-type (WT), phyB, phyA (cultured in absence or presence of GA), and phyA/aba2 embryos $50 \mathrm{~h}$ after seed imbibition. The histogram shows the length of embryos from apical to root meristem. $(C$, panel $a)$ The left diagram shows the procedure to culture coatless embryos. (Panel b) The right diagram depicts the procedure to isolate embryos from cultured seeds. Relative GA3ox1/2 and NCED6/9 mRNA accumulation in wild-type (WT), phy $A$, and phy $B$ embryos $28 \mathrm{~h}$ after imbibition obtained as shown in the diagrams. Relative transcript levels were normalized to those of UBQ11. (D) Time course of phyA and phyB protein accumulation in wild-type (Col) seeds before an early FR pulse $(4 \mathrm{~h})$ and thereafter upon transfer to darkness (12 h, $24 \mathrm{~h}$, and $48 \mathrm{~h}$ ). phy $A / B$ double mutants seeds $(A / B)$ were used as negative control for antibody specificity. $(E)$ phyA-dependent germination was assessed after a second FR pulse applied $12 \mathrm{~h}$ (gray bar) or $48 \mathrm{~h}$ (black bar) after seed imbibition. Germination frequency (percent) was scored $120 \mathrm{~h}$ after seed imbibition.

germination results from low GA synthesis and high ABA levels. Indeed, phy $A$ and phy $A C D E$ embryonic growth was restored upon addition of GA, and phyA/aba coatless embryos germinated (Fig. 1B; Supplemental Fig. 1B).

phyA accumulation has been shown to be low in dry seeds relative to that of phyB and to rise in dark-treated seeds upon imbibition (Shinomura et al. 1996). Low phy $A$ accumulation was invoked to explain lack of phyAdependent germination at the early stages of seed imbibition. We re-evaluated this model under conditions leading to phyA-dependent germination rather than in dark-treated seeds. phyA accumulation was very low prior to a first FR pulse; however, phyA levels rapidly increased, reaching plateau levels $12 \mathrm{~h}$ after seed imbibition (Fig. 1D). Despite similar phyA levels at $12 \mathrm{~h}$ and $48 \mathrm{~h}$, a second FR pulse applied at $12 \mathrm{~h}$ barely stimulated germination relative to a pulse applied at $48 \mathrm{~h}$, inconsistent with a model where phyA accumulation alone determines the potential for phyA-dependent germination (Fig. 1E).

\section{All phytochromes signal in the embryo to promote germination}

We further studied coatless embryonic germination under conditions leading to phyB-dependent germination, such as after FR/R pulses. Strikingly, FR/R-treated phyB coatless cultured embryos germinated, unlike phyB seeds, and expressed high GA3ox1/2 and low NCED6/9 expression relative to embryos dissected from $p h y B$ seeds treated in the same manner (Fig. 2A,B). Thus, the seed coat also plays an essential role to prevent phyB seed germination. Germination of FR/R-treated phy $B$ coatless embryos could be due to any other phytochrome signaling in the embryo (Poppe and Schafer 1997; Hennig et al. 2002; Franklin et al. 2003; Dechaine et al. 2009; Strasser et al. 2010). Indeed, only FR/Rtreated phy $A B C D E$ coatless embryos failed to germinate, unlike phy $A C D E$ or $p h y B C D E$ embryos, and had low GA3ox1/2 expression and high NCED6/9 expression relative to phy $A C D E$ or $p h y B C D E$ embryos, consistent with the notion that their lack of germination results from low GA and high $\mathrm{ABA}$ levels, respectively (Fig. 2A,C). Taken together, these experiments show that all phytochromes are able to signal in the embryo to promote embryonic growth.

In summary, these observations do not support the usually accepted notion that only phyA levels determine the potential for phyA-dependent germination. Instead, it appears that phyA is potentially able to promote germination early upon seed imbibition, but this phyA-dependent 
A

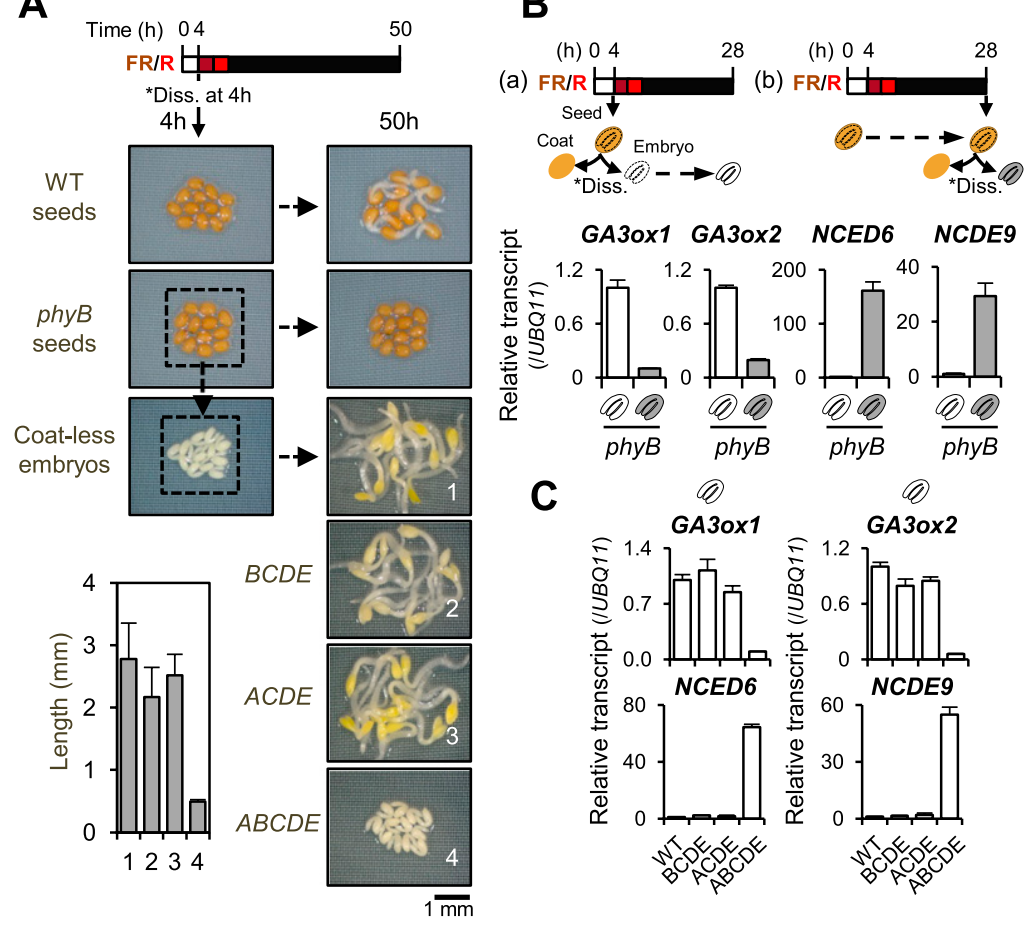

Figure 2. The seed coat is essential for phyBdependent control of seed germination. All phytochromes promote coatless embryo germination. $(A)$ Seeds of the indicated genotype were manipulated as in Figure 1B except that the FR pulse was followed by a R pulse. (B) Relative GA3ox1/2 and NCED6/9 mRNA accumulation in FR/R-treated phy $B$ embryos isolated as in Figure 1C. $(C)$ Relative GA3ox1/2 and NCED6/9 mRNA accumulation in FR/R-treated cultured coatless embryos of the indicated genotype and obtained as depicted in the left diagram in $B$, panel $a$. germination activity is prevented by the seed coat. Furthermore, the seed coat exerts a repressive activity that is necessary to block germination upon FR-dependent phyB inactivation or when phyB is absent, as in $p h y B$ mutants. Finally, seed coat removal reveals that all phytochromes are able to signal in the embryo to promote germination.

\section{A SCBA shows that phyB signals in the endosperm to control synthesis and release of $A B A$}

Previous work showed that the endosperm releases ABA to prevent dormant seed germination (Lee et al. 2010). Using a SCBA, we addressed the hypothesis that phytochromes similarly control ABA synthesis and release from the endosperm to control embryonic growth (Fig. 3A; Lee et al. 2010).

A SCBA was assembled with dissected wild-type embryos laid on a bed of dissected wild-type seed coats. Strikingly, wild-type embryos did not germinate after a FR pulse irradiating both embryos and seed coats (Fig. 3B). Germination repression did not require direct contact between seed coats and embryos, since germination repression was maintained in the presence of a nylon filter separating seed coats and embryos (Fig. 3C). Germination arrest specifically required FR irradiation, since embryos germinated when the FR pulse was followed by a second $\mathrm{R}$ pulse irradiating both embryos and seed coats (Fig. 3B). These observations show that the SCBA can be used as a genetic in vitro model to study early FR-dependent germination repression. We next asked whether R-dependent germination in a SCBA involves the endosperm. Indeed, and remarkably, seed coats irradiated by FR/R pulses could not repress the growth of embryos irradiated with a FR pulse (Fig. 3C).

Previous work showed that isolated dormant seed coats synthesize and release ABA, while aba2 seed coats, unable to synthesize ABA, failed to block embryonic growth in a SCBA (Lee et al. 2010). Similarly, a SCBA with aba2 seed coats did not repress wild-type embryo germination after a FR pulse (Fig. 3D). In contrast, wild-type seeds coats could repress aba2 embryo germination, although not as markedly (Fig. 3D). This indicates that ABA synthesis is required in both the endosperm and embryo for full embryonic arrest in a SCBA. We tested the capacity of dissected seed coats to synthesize and release ABA in response to light cues. In mature dry seeds, ABA levels were shown to be initially very elevated and to drop markedly within the first $24 \mathrm{~h}$ after imbibition, even after a FR pulse and in absence of GA synthesis (Piskurewicz et al. 2009). Indeed, blockade of seed germination is determined by the capacity of imbibed seeds to sustain high ABA accumulation over time; i.e., beyond the first $24 \mathrm{~h}$ after imbibition (Piskurewicz et al. 2008, 2009; Lee et al. 2010). Thus, to prevent carryover of ABA present in the mature seed from masking changes in ABA accumulation upon imbibition, seed coat material was dissected $24 \mathrm{~h}$ after imbibition of wild-type seeds in darkness (see the Materials and Methods). Thereafter, seed coats were treated with a FR pulse or FR/R pulses and cultured for an additional $24 \mathrm{~h}$ in darkness. Figure $3 \mathrm{E}$ shows absolute ABA levels measured in seed coat material and in the surrounding medium (see the Materials and Methods). Strikingly, and consistent with the notion that the seed coat is a light-sensitive tissue releasing ABA in response to FR, several hundred-fold higher ABA levels were found 
Lee et al.

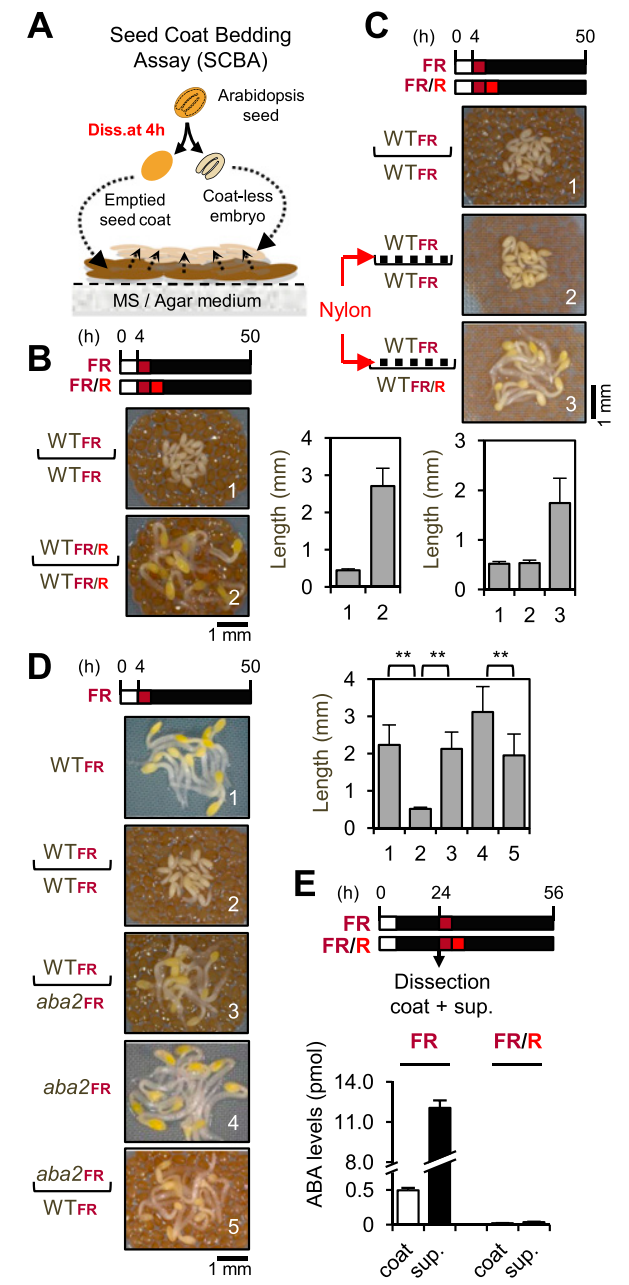

Figure 3. The seed coat acts as a light-sensitive tissue inhibiting embryonic growth in response to FR. $(A)$ Procedure for assembling a SCBA. (B) SCBAs: Embryos and seed coats were dissected $4 \mathrm{~h}$ after seed imbibition, and coatless embryos were laid on a layer of dissected seed coats before light irradiation and transfer to darkness. Seed coats and embryos were subjected to the same light treatment as shown: FR pulse (FR) or FR pulse followed by R pulse (FR/R). The histogram shows the length of embryos from apical to root meristem. $(C)$ Same as in $B$ except that a nylon filter, unable to block light irradiation, separates the seed coats and embryos. In panel 3, seed coats and embryos were subjected to different light treatment as indicated. Length measurements as in $B$. (D) SCBAs with FR-treated wild-type (WT) or $a b a 2$ seed coats and with wild-type or $a b a 2$ embryos, as indicated. Asterisks indicate a statistically significant difference based on a two-tailed $t$-test $(P<0.01)$. (E) Seed coats were dissected from wild-type seeds $24 \mathrm{~h}$ after imbibition and incubated in MS medium prior to irradiation with FR or FR/R pulses followed by $32 \mathrm{~h}$ of incubation in darkness (Material and Methods). Histograms show ABA levels (in picomoles) measured in dissected seed coat materials (coat) and seed coats incubation medium. (sup.) Supernatant.

in FR-treated dissected wild-type seed coats and their surrounding medium, which contained the majority of the measured $\mathrm{ABA}$, than in the corresponding FR/R-treated material.
We observed above that the seed coat plays an essential role to prevent $p h y B$ seed germination (Fig. 2A). Furthermore, seed coats block germination in a SCBA in response to FR but not in response to $\mathrm{R}$ (Fig. $3 \mathrm{C}$ ). These observations indicate that phyB plays a predominant role in the endosperm, where absence of phyB or phyB inactivation by FR leads to ABA synthesis and release from the endosperm toward the embryo. Consistent with this view, phyB mutant seed coats irradiated by FR/R pulses could still repress the germination of wild-type embryos irradiated with a FR pulse in a SCBA, unlike wild-type or phy A seed coats (Fig. 4A; Supplemental Fig. 2A). In contrast and furthermore, consistent with the hypothesis, phyB/aba2 mutant seed coats irradiated by FR/R pulses could not repress the germination of wild-type embryos in a SCBA (Fig. 4A; Supplemental Fig. 2A). Furthermore, phyB seed coats dissected from FR/R-treated phy $B$ seeds retained high ABA levels relative to wild-type or phy $A$ seed coats dissected from similarly treated seeds (Fig. 4B). As expected, wild-type, phy $A$, and $p h y B$ dissected seed coats isolated from FR-treated wild-type, $p h y A$, and $p h y B$ seeds had at least fivefold more ABA levels than seed coats isolated from FR/R-treated wild-type or phyA seeds (Fig. 4B).

In summary, these results clearly indicate that (1) a SCBA maintains FR-dependent germination repression, which requires $\mathrm{ABA}$ synthesis and release from the seed coats; (2) seed coats display autonomous capacity to release ABA in response to FR but not in response to R; and (3) phyB signals in the endosperm to control synthesis and release of $\mathrm{ABA}$ to block germination.

\section{Tissue- and phytochrome-specific signaling in cultured seeds}

So far, we cultured dissected seed coat and embryonic material to propose that phyA and phyB specifically signal in the embryo and endosperm, respectively. We wished to confirm this notion directly in seeds by measuring phyA- and phyB-dependent gene expression in noncultured seed coat and embryo material; i.e., directly isolated from cultured intact seeds. Seed coats dissected from FR/R-treated $p h y B$ seeds had low GA3ox1/2 and high NCED6/9 expression relative to wild-type and phy $A$ coats (Fig. 4C). In contrast, seed coats isolated from FR-treated wild-type, phy $A$, and phyB seeds had similar GA3ox1/2 and NCED6/9 expression levels (Fig. 4C).

These results confirm that phyB, unlike phyA, specifically signals in the endosperm at early time points upon seed imbibition. However, phyA protein was not detectable in seed coats at early time points (Supplemental Fig. 2B). To rule out the possibility that lack of phyA signaling in the endosperm is due to low phyA accumulation, we examined phyA-dependent expression in seed coats dissected from seeds after a later second FR pulse that triggers phyA-dependent germination. Consistent with our hypothesis, wild-type and phyA seed coats maintained similarly low GA30x1/2 expression and high NCED6/9 expression despite the occurrence of phyAdependent germination (Fig. 4D). Furthermore, wild-type and phy $A$ seed coats retained high ABA levels in both FR- 
A
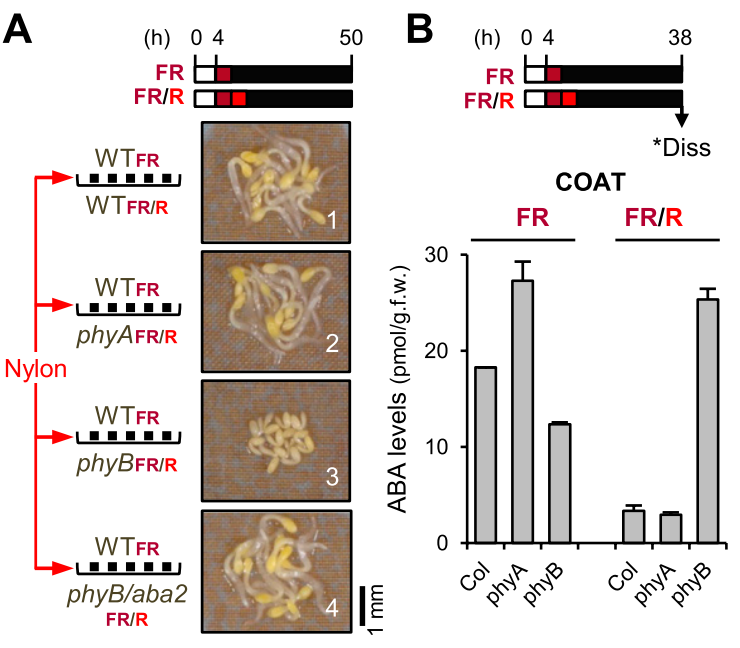

C
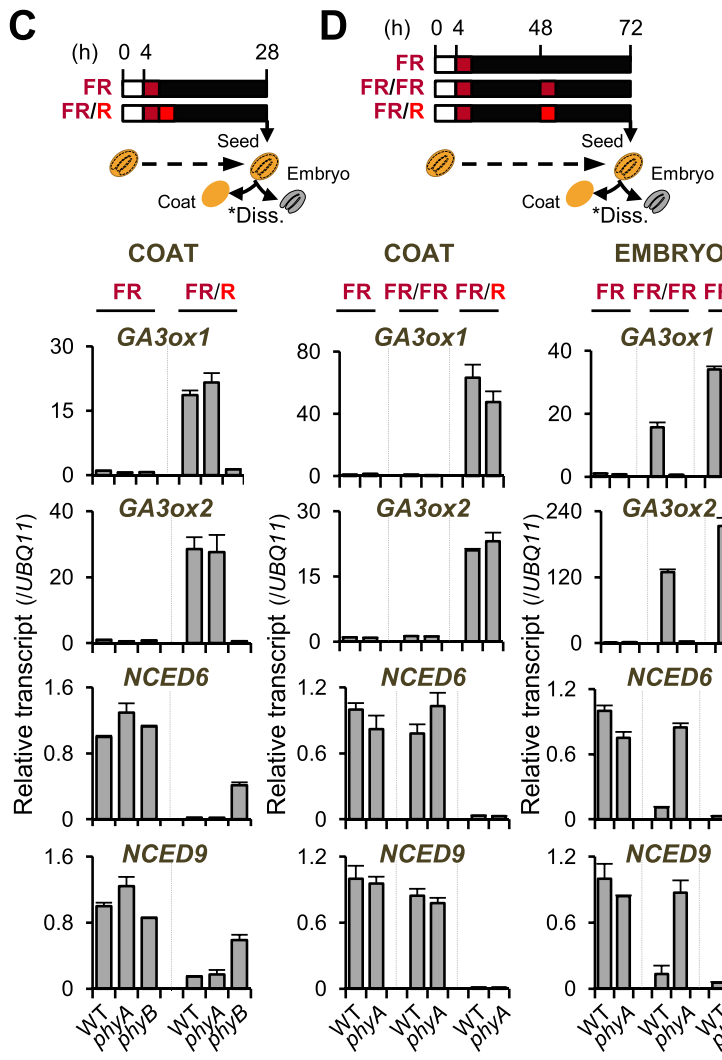

COAT
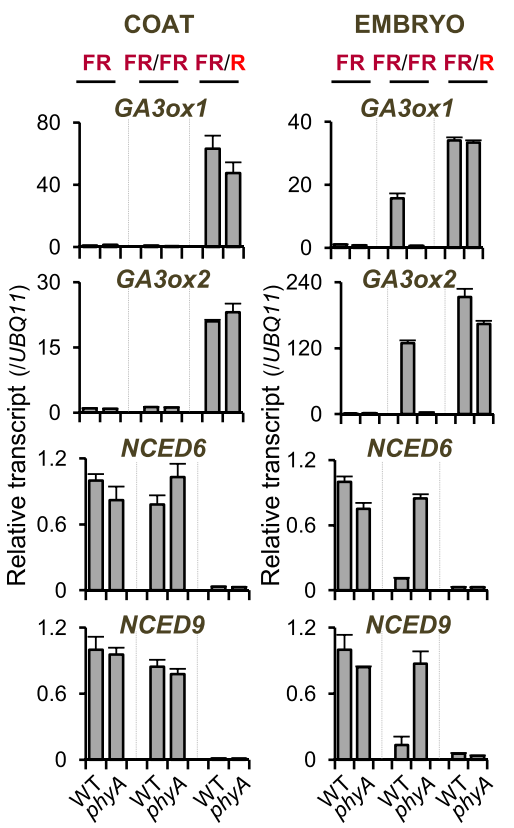

Figure 4. phyB inactivation or phyB absence in the seed coat requires $\mathrm{ABA}$ synthesis to block embryonic growth. phyB specifically signals in the endosperm in response to R. phyA specifically signals in the embryo in response to a second late FR pulse. (A) SCBAs with FR-treated wild-type (Col) embryos and FR/R-treated wild-type (WT), phyA, phyB, and phyB/aba2 seed coats. SCBAs are pictured $50 \mathrm{~h}$ after imbibition. $(B)$ Endogenous ABA levels in seed coats dissected $38 \mathrm{~h}$ after seed imbibition from FR- and FR/R-treated wild-type, phy $A$, and phy $B$ seeds as shown. (C) Relative GA3ox1/2 and NCED6/9 mRNA accumulation in wild-type (WT), phy $A$, and phyB seed coats isolated from FR- and FR/R-treated seeds $28 \mathrm{~h}$ after seed imbibition as shown. (D) Relative GA3ox1/2 and NCED6/9 mRNA accumulation in wild-type and phyA seed coats and embryos isolated from FR-, FR/FR-, and FR/R-treated seeds $72 \mathrm{~h}$ after seed imbibition as shown. ( ${ }^{\star}$ Diss.)Dissection. or FR/FR-treated seeds (Supplemental Fig. 2C). In contrast and strikingly, wild-type embryos had high GA3ox1/ 2 expression and low NCED6/9 expression relative to phy $A$ embryos (Fig. 4D). ABA levels dropped by $60 \%$ in wild-type embryos, whereas they remained high in $p h y A$ embryos (Supplemental Fig. 2C). Taken together, these data show that phyA specifically signals in the embryo and that phyB specifically signals in the endosperm.

\section{phyB signaling in the endosperm overrides phy $A$ signaling in the embryo: the role of $A B A$}

Expectedly, wild-type embryo growth arrest in a SCBA was associated with low $G A 30 x 1 / 2$ expression relative to germinated embryos (Fig. 3D; Supplemental Fig. 3A). Furthermore, when coatless cultured wild-type embryos were directly treated with ABA, they expectedly did not germinate and had low $G A 30 \times 1 / 2$ expression relative to untreated controls (Supplemental Fig. 3B). This strongly indicates that endospermic ABA directly participates in blocking phyA-dependent stimulation of GA3ox1/2 expression in the embryo.

Taken together, our results so far show that phyB in effect converts the endosperm into a light-sensitive tissue mediating early light-dependent control of seed germination. When phyB is in its inactive Pr form, as in FR-treated seeds, or when phyB is absent, as in phyB mutants, the endosperm releases $\mathrm{ABA}$, which is released toward the embryo to repress phyA-dependent GA3ox $1 / 2$ expression, thus blocking embryo germination as a result of high ABA levels resulting from low GA synthesis. When phyB is in its active Pfr form, as in R-treated seeds, ABA release is reduced, thus allowing germination to occur.

\section{ABA-dependent responses weaken over time, allowing phyA-dependent germination}

This model emphasizes the central role of ABA to counteract phyA-dependent signaling in the embryo. Previous work showed that ABA- and ABI5-dependent growth arrest weakens over time; indeed, exogenous ABA only represses germination within a limited time window upon seed imbibition (Lopez-Molina et al. 2001). Here, we observed that NCED6/9, $A B I 3$, and $A B I 5$ expression significantly decreased over time after the first FR pulse in whole seeds but also in seed coats and embryos dissected from whole seeds (Fig. 5A,B; Supplemental Fig. 4B). Furthermore, endogenous ABA levels in FR-treated wildtype seeds measured $192 \mathrm{~h}$ after imbibition were $50 \%$ lower relative to those measured at $18 \mathrm{~h}$ (Fig. 5C). In contrast, GA3ox1/2 expression remained invariably low, suggesting that the observed weakening in ABA-dependent responses does not result from a derepression in GA synthesis (Supplemental Fig. 4A,B). Weaker ABA-dependent responses could underlie phyA-dependent germination after a second FR pulse at later time points. We tested this possibility using abi5 mutants or wild-type seeds in which endogenous ABA synthesis was repressed by norflurazon treatment. 
Lee et al.

A

B

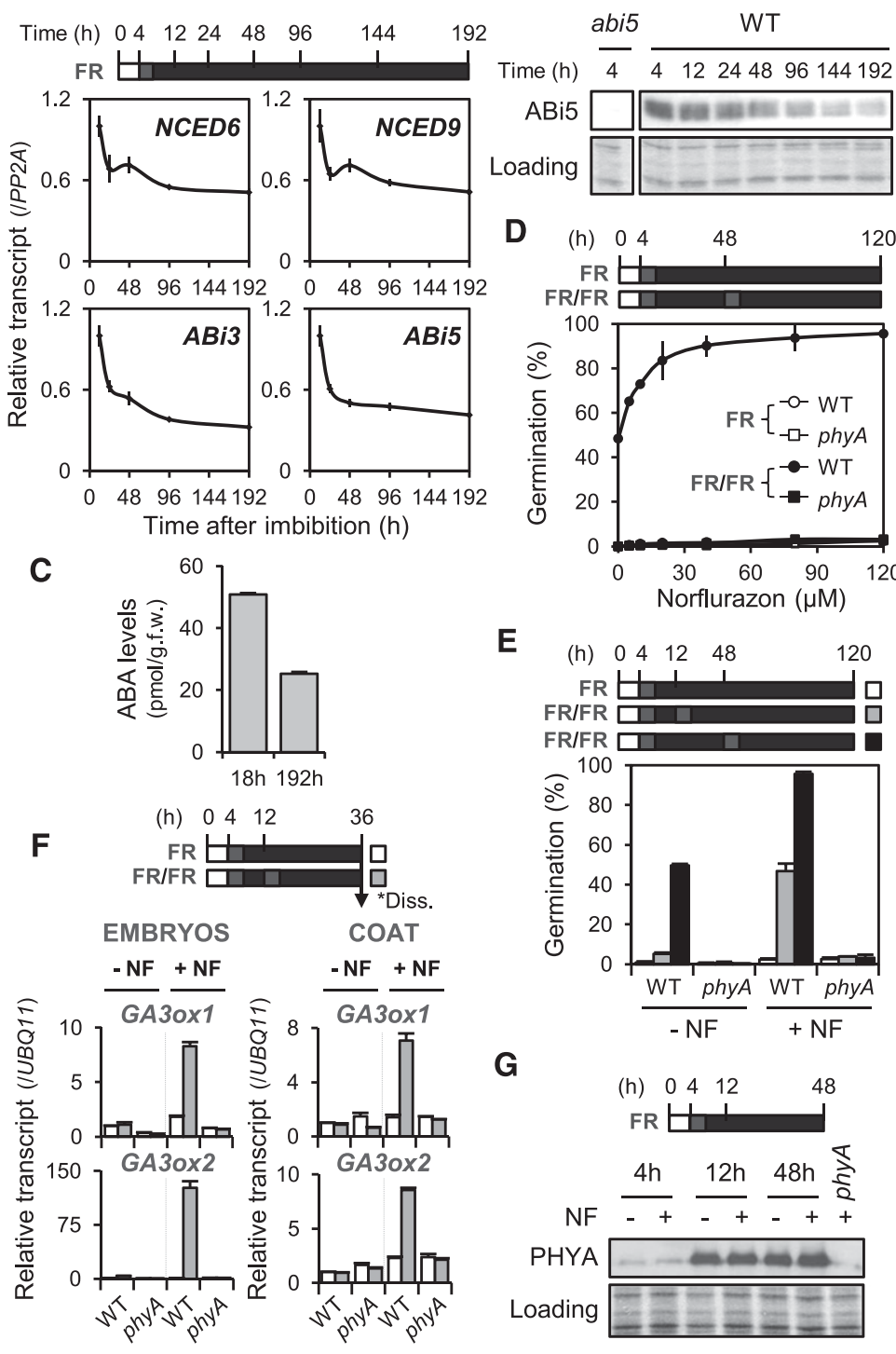

Figure 5. Weaker ABA-dependent responses over time promote phyA-dependent germination. $(A)$ Time course of NCED6/9, ABI3, and ABI5 mRNA accumulation in FR-treated wild-type (Col) seeds. (B) Time course of ABI5 protein accumulation in wild-type (WT) seeds treated as in A. abi5 mutant seed material harvested $4 \mathrm{~h}$ after imbibition is used to control for ABI5 antibody specificity. $(C)$ Endogenous ABA levels in wild-type seeds treated as in $A 18 \mathrm{~h}$ and $192 \mathrm{~h}$ after seed imbibition. $(D)$ phyA-dependent germination assays with wild-type (WT) and phyA seeds in the presence of various norflurazon concentrations. (E) Wild-type (WT) and phy $A$ germination after a second FR pulse applied $12 \mathrm{~h}$ or $48 \mathrm{~h}$ after seed imbibition in the absence or presence of norflurazon (NF, $120 \mu \mathrm{M})$. (F) Relative GA3ox1/2 mRNA accumulation in seed coats and embryos isolated from wild-type (WT) and phyA seeds after a second FR pulse applied $8 \mathrm{~h}$ after a first early FR pulse in the absence or presence of norflurazon (NF, 80 $\mu \mathrm{M})$. (G) phyA protein accumulation in FR-treated seeds at the indicated times after imbibition in the absence or presence of norflurazon (NF, $120 \mu \mathrm{M})$. ( ${ }^{\star}$ Diss.) Dissection.
Seeds were cultured in presence of a range of norflurazon concentrations that maintain germination arrest after an early first FR pulse. Strikingly, the lowest norflurazon concentration used (5 $\mu \mathrm{M})$ was sufficient to stimulate germination by $35 \%$ in a phyA-dependent manner after a second FR pulse (Fig. 5D). Furthermore, a FR pulse applied $12 \mathrm{~h}$ after the first FR pulse triggered $50 \%$ germination of norflurazon-treated wild-type seeds but not that of phy $A$ mutants (Fig. 5E). Germination was associated with higher phyA-dependent $G A 30 x 1 / 2$ expression in the embryo, as expected, but also, significantly, in the seed coat. In each case, no substantial changes in phyA protein accumulation could be observed (Fig. 5F,G).

We previously reported that abi5 mutant seeds do not germinate in response to an early FR pulse, since ABI5 mediates a subset of ABA-dependent responses blocking germination (Piskurewicz et al. 2009). However, a second FR pulse applied $12 \mathrm{~h}$ after seed imbibition markedly stimulated seed germination and $G A 30 \times 1 / 2$ expression in abi5 mutant seeds, unlike in wild-type seeds (Supplemental Fig. 4C,D).

Taken together, these observations support the model that a gradual decrease over time in ABA-dependent responses enables phyA-dependent signaling and germination.

\section{RGL2 signals in the endosperm; GAI and RGA signal in the embryo}

A previous report showed that PIF1, SOM, RGL2, GAI, and $R G A$ are expressed in both the endosperm and the embryo (Penfield et al. 2006). This prompted us to ask whether these phytochrome signaling components exhibit tissue-specific activities.

The DELLA factors RGA, GAI, and RGL2 redundantly maintain high levels of ABA (Piskurewicz et al. 2008, 2009). gai/rga seed coats retained the ability to block wildtype embryo germination in a SCBA (Fig. 6A). In contrast, rgl2 seed coats were unable to arrest wild-type germination 

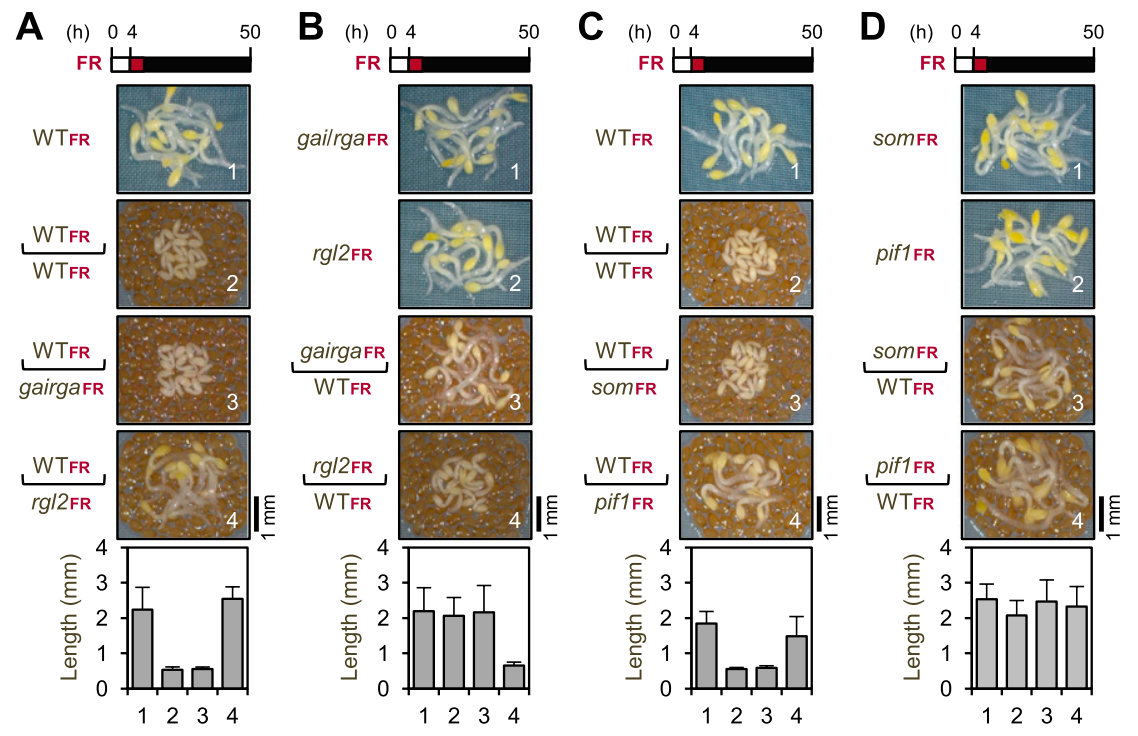

Figure 6. RGL2 and PIF1 signal in the endosperm, and PIF1, SOM, GAI, and RGA signal in the embryo. (A) SCBAs with FRtreated wild-type (Col) embryos on a bed of FR-treated wild-type (WT), gai/rga, and rgl2 seed coats. SCBAs are pictured $50 \mathrm{~h}$ after imbibition. The histogram shows the length of embryos from apical to root meristem. $(B)$ SCBAs as in $A$ using gai/rga or rgl2 embryos and wild-type (WT) seed coats. (C) SCBAs as in $A$ using wild-type (WT) embryos and som or pif1 seed coats. (D) SCBAs as in $A$ with som or pif1 embryos and wild-type (WT) seed coats.
(Fig. 6A). However, wild-type seed coats were able to block rgl2 germination but not that of gai/rga embryos (Fig. 6B). These results therefore indicate that RGL2 specifically signals in the seed coat, whereas GAI and RGA signal in the embryo. Indeed, seed coats dissected from FR-treated rgl2 seeds had high GA3ox1/2 and low NCED6/9 expression relative to wild-type seed coats (Supplemental Fig. 5A). However, GA3ox1/2 and NCED6/ 9 expression was similar in embryos dissected from $\mathrm{rgl}$ and wild-type seeds (Supplemental Fig. 5A). The same approach with seed coat and embryonic material isolated from FRtreated gai/rga seeds confirmed that GAI and RGA control GA3ox1/2 and NCED6/9 expression in the embryo but not in the seed coat (Supplemental Fig. 5A).

\section{PIF1 signals in the endosperm and embryo; SOM signals in the embryo}

Previous reports have proposed that PIF1 represses germination, notably by stimulating the expression of SOM. Wild-type and som germination was similarly repressed after an early FR pulse (data not shown), which is not consistent with previous reports (Kim et al. 2008). This discrepancy could be due to our use of a different FR irradiation protocol or seed production conditions. Testa rupture is not visible in FR-treated som seeds, unlike pif1 seeds, suggesting that SOM does not signal in the seed coat, unlike PIF1 (Supplemental Fig. 5B). Indeed, som seed coats retained the ability to block wild-type embryo germination in a SCBA, unlike pif1 mutant seed coats (Fig. 6C). Furthermore, seed coats isolated from FRtreated pif1 seeds had high GA3ox1/2 and low NCED6/ 9 expression relative to seed coats isolated from wild-type or som seeds (Supplemental Fig. 5C). It is significant to note that $S O M$ expression was low in pif1 mutant seed coats, consistent with the previous reports (Supplemental Fig. 5D; Kim et al. 2008). Concerning the role of PIF1 and SOM in the embryo, wild-type seed coats were unable to prevent pif1 and som embryo germination in a SCBA, suggesting that both PIF1 and SOM signal in the embryo (Fig. 6D). Indeed, som and pif1 embryos dissected from FR-treated som and pif1 seeds had high GA3ox1/2 and low NCED6/9 expression relative to wild-type embryos, consistent with previous reports using whole seeds (Supplemental Fig. 5E; Oh et al. 2007; Kim et al. 2008).

\section{$A B A$ and $A B I 5$ regulate the expression of $G A$ and $A B A$ metabolic genes and light signaling genes}

We previously identified a positive feedback loop involving ABA and RGL2 whereby ABA stimulates RGL2 mRNA levels and RGL2 promotes ABA synthesis (Piskurewicz et al. 2008). The present observations suggest that endospermic ABA sustains an additional positive feedback loop by repressing GA3ox1/2 gene expression in the embryo.

This suggested that ABA may play a more general role to feedback-regulate the expression of key components of phytochrome signaling pathways whose final output is to control germination by controlling ABA levels. We compared the expression of light signaling genes in FR-treated ga1 versus ga1/aba2 seeds, seed coats, and embryos and in FR-treated ga1 versus ga1/abi5 seeds, seed coats, and embryos $28 \mathrm{~h}$ and $72 \mathrm{~h}$ after seed imbibition (Fig. 7A; Supplemental Fig. 6A,B; data not shown). The use of a GA synthesis-deficient ga1 background allowed the elimination of an influence on gene expression resulting from changes in GA levels. The results shown in Figure 7A and Supplemental Figure 6, A and B, support the notion of a widespread ABA positive feedback mechanism that stimulates ABA synthesis by acting not only on GA and ABA metabolic genes, but also on that of key light signaling genes.

\section{ABI5 interacts with promoter sequences in vivo}

We explored whether ABI5 could bind in vivo with the promoter sequences of these genes by combining chromatin immunoprecipitation (ChIP) with massively parallel DNA sequencing (ChIP-seq). ChIPs using antibodies 
Lee et al.

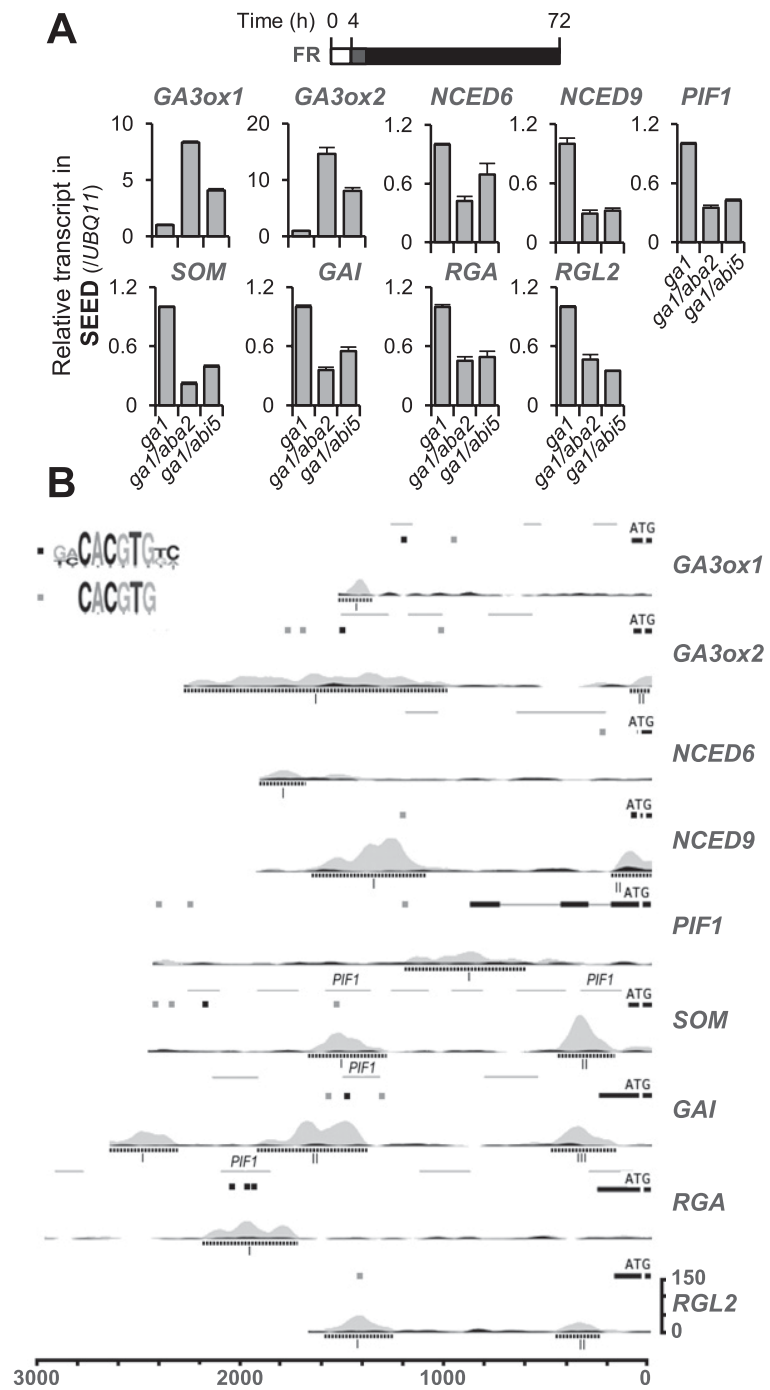

Figure 7. $\mathrm{ABA}$ and $\mathrm{ABI} 5$ regulate expression of $\mathrm{GA}$ and $\mathrm{ABA}$ metabolic genes and light signaling genes, which is associated with ABI5 binding in vivo to promoter DNA sequences. $(A)$ Relative GA3ox1/2, NCED6/9, PIF1, SOM, GAI, RGA, and RGL2 mRNA accumulation in FR-treated ga1, ga1/abi5, and ga1/aba2 seeds $72 \mathrm{~h}$ after imbibition. (B) Distribution of DNA sequencing reads present upstream of GA and ABA metabolic genes and light signaling genes in Input (black) and Exp (gray) ChIP precipitates. The vertical scale shown only for RGL2 indicates the occupancy strength and is the same for all genes. The bottom horizontal scale indicates the distance relative to the translation start site (ATG) of each indicated gene. Dashed lines encompass DNA regions with statistically higher ABI5 occupancy and are labeled with roman numerals (I, II, and III). Horizontal gray lines in GA3ox1, GA3ox2, NCED6, SOM, GAI, and $R G A$ indicate DNA regions where PIF1 binding was monitored in Oh et al. (2007) and Kim et al. (2008). "PIF1" above the gray lines indicates significant PIF1 binding. (G/T) (A/C)CACGTG(T/G)(C/A) and CACGTG (G-box) DNA sequences are shown as black and gray squares, respectively.

against hemagglutinin (HA) were performed on estradioland ABA-treated abi5-4 (Cont) mutants and abi5-4 mutants transformed with an estradiol-inducible promoter driving the expression of $H A$-tagged ABI5 (abi5-4/ind::
HA-ABI5; Exp). Genomic DNA isolated from abi5-4/ ind::HA-ABI5 material was sequenced and used as a DNA reference sequence (Input). Statistical analysis of the whole DNA library sequencing data set revealed that the sequence $(\mathrm{G} / \mathrm{T})(\mathrm{A} / \mathrm{C}) \mathrm{CACGTG}(\mathrm{T} / \mathrm{G})(\mathrm{C} / \mathrm{A})$, containing a G-box (CACGTG), is overrepresented in DNA domains with a high ABI5 occupancy (see the Materials and Methods). Figure 6B compares the distribution of sequencing reads present in Input (black) and Exp (gray) ChIP precipitates that match promoter sequences of GA3ox1/2, NCED6/9, SOM, GAI, RGA, and RGL2. DNA promoter regions with higher ABI5 occupancy are underlined (Fig. 7B), and their statistical significance was analyzed (Fig. 7B; Supplemental Fig. 6C). Significant binding $(P<0.05)$ was found in GA3ox1 (peak I), GA3ox2 (peaks I and II), NCED9 (peak I), SOM (peaks I and II), GAI (peaks I, II, and III), RGA (peak I), and RGL2 (peaks I and II). No significant binding $(P>0.05)$ was found in NCED6 and PIF1. Five out of six peaks with the strongest occupancy (fold change, $>10$ ) were associated with the core sequence $(\mathrm{G} / \mathrm{T})(\mathrm{A} / \mathrm{C}) \mathrm{CACGTG}(\mathrm{T} / \mathrm{G})(\mathrm{C} / \mathrm{A})$, a G-box, or both, whereas weaker peaks (fold change, $<10$ ) associated two times out of six (Fig. 7B; Supplemental Fig. 6C), consistent with the results of the whole data set statistical analysis (see the Materials and Methods). It is worth noting that in the case of GA3ox2, wide DNA distribution of ABI5 occupancy (peak I) coincided with four ABI5-binding motifs distributed along the DNA region occupied by ABI5. These data show that with the exception of PIF1 and NCED6, ABI5 can be associated in vivo with at least one DNA domain of all of the examined promoter sequences.

Previous ChIP experiments showed that PIF1 is associated in vivo with DNA regions of GAI, RGA, and SOM promoters containing G-box elements. However, PIF1 was not found to be associated with GA3ox1/2 or NCED6 promoters (Oh et al. 2006). For the sake of comparison, we included in Figure 7B the DNA regions where PIF1 occupancy was previously analyzed. Interestingly, ABI5 DNA occupancy regions overlapped with the G-box-containing DNA regions occupied by PIF1 in the SOM, GAI, and $R G A$ promoters, indicating that PIF1 and ABI5 could bind the same G-box motifs to regulate transcription. Interestingly, in the case of SOM, both PIF1 and ABI5 (peak II) are associated with the same DNA region, which lacks a G-box domain but contains a CACATG motif that can interact with PIF1 in vitro.

Together with the gene expression data shown in Figure 7A, these ChIP-seq data render plausible the idea that ABI5 could directly participate in regulating the transcription in all examined genes, with the exception of PIF1 and NCED6.

\section{Discussion}

The developmental switch from phyB- to phyAdependent germination: a switch from early $A B A$ dominance to later GA dominance

It is worth reminding that GA negatively regulates $A B A$ levels and that only $\mathrm{ABA}$ levels determine the germination 
potential of a seed (Piskurewicz et al. 2008, 2009). Further understanding how phytochromes control germination requires understanding how their signaling intermediatesPIF1, SOM, GAI, RGA, and RGL2-regulate changes in GA and ABA synthesis. However, it follows from this work that it will also require understanding how endospermic ABA blocks phyA signaling in the embryo after an early FR pulse. The Pfr active form of phyA destabilizes PIF1, which blocks germination by stimulating $S O M, G A I$, and RGA expression. These factors repress GA3ox1/2 and stimulate NCED6/9 expression, respectively (Fig. 6; Supplemental Fig. 5). The data presented here suggest that $\mathrm{ABA}$ overrides phyA signaling through ABI5, which, as PIF1, negatively regulates germination. We show that PIF1 and ABI5 share a number of target genes implicated in the repression of seed germination (Figs. 7A, 8 [left part of model]; Supplemental Fig. 6A,B). This mechanism could compensate for PIF1 destabilization in the embryo when the endosperm synthesizes high ABA levels (e.g., in the shade) (Fig. 8, left part of model). Further compensation could involve ABA- and ABI5dependent stimulation of PIF1 mRNA accumulation. However, ABI5 could also directly target GA and ABA metabolism by repressing GA3ox $1 / 2$ expression and stimulating NCED6/9 expression, respectively, thus lowering GA levels and further stimulating $\mathrm{ABA}$ levels in embryos (Figs. 3D, 8 [model]; Supplemental Figs. 3A,B, $6 \mathrm{~A}, \mathrm{~B})$.

Besides the question of how ABA interferes with phyA signaling, the conclusion that $\mathrm{ABA}$ represses GA synthesis may help understanding how the seed irreversibly switches from FR-dependent repression of phyB-dependent germination to FR-dependent stimulation of phyA-dependent germination. Once the weakening of the ABA-dependent

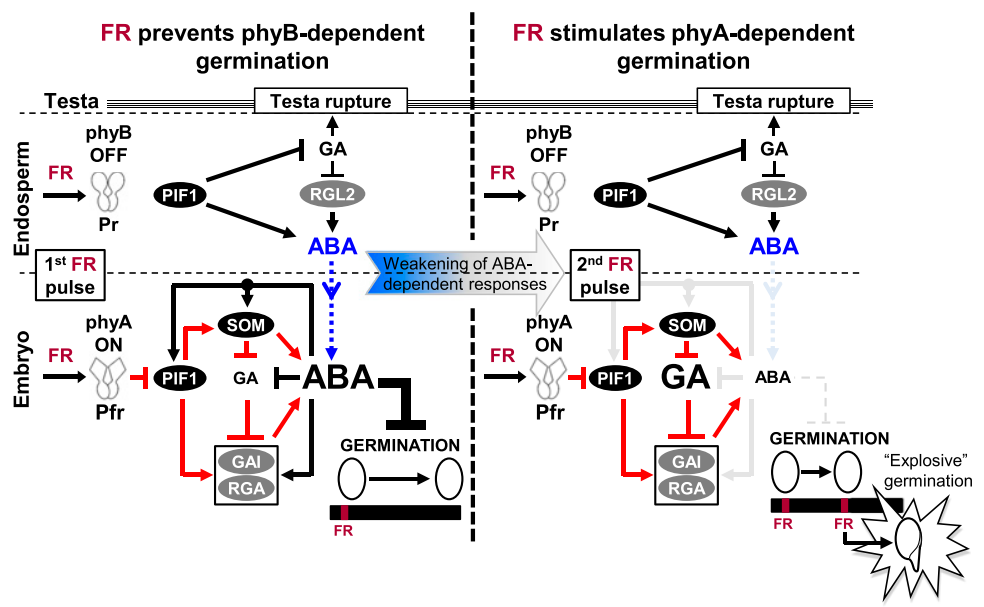
tion; and (5) GAI- and RGA-dependent stimulation of ABA synthesis. Endospermic ABA is released toward the embryo (blue line) and joins the pool of ABA synthesized in the embryo (shown as "ABA" in black). ABA overrides (black lines) phyA signaling by repressing GA3ox1/2 (GA) expression and stimulating PIF1, SOM, RGA, and GAI expression, thus further repressing GA3ox1/2 (GA) expression and stimulating NCED6/9 (ABA) expression. As a result, ABA dominates to repress germination. (Right) A second later FR pulse maintains in the endosperm the situation described in the left part of the figure, since phyB remains inactive. However, ABA-dependent responses become weaker over time, allowing the second FR pulse to stimulate and repress embryonic GA and ABA synthesis gene expression, respectively, in a phyA-dependent manner (Fig. 4D, 5). GA accumulation is expected to further weaken ABA-dependent responses by repressing GAI and RGA accumulation. This leads to "explosive" germination, as embryonic growth occurs in a context of unruptured testa. responses is initiated, a second FR pulse will stimulate phyA-dependent GA3ox1/2 expression, thus stimulating GA accumulation, which will lead to further decay in dependent responses blocking phyA signaling (Fig. 8, right part of model).

The mechanisms responsible for the weakening of ABA-dependent responses over time remain unknown. In this respect, it was previously shown that ABA-dependent repression of embryonic growth is short-lived, occurring within a limited time window upon seed imbibition (Lopez-Molina et al. 2001). It was proposed that stress would trigger a protective and ABA-dependent growth arrest that would maintain the stress-resistant embryonic state. However, this ABA-dependent growth arrest is irreversibly lost once the plant is developmentally engaged toward its vegetative phase, where it will mount ABA-dependent stress responses more appropriate for vegetative growth (Lopez-Molina et al. 2002). It is tempting to speculate that the same mechanism that dependent germination after a second FR pulse.

The role of endospermic release of $\mathrm{ABA}$ to modulate phyA-dependent responses further confirms the importance of $\mathrm{ABA}$ as the decisive factor determining the germination potential of the seed. From an evolutionary perspective, it may be advantageous for a plant under light stress to sustain high ABA levels for extended periods of time by means of the self-reinforcing feedback nism proposed above. However, once the seed is growth indefinitely. Inty disadvantageous to prevent active process that may exhaust food stores required to complete germination. Furthermore, the advantages of $\mathrm{ABA}$ accumulation and thus further weakening of ABA-

Figure 8. Model for phyB- and phyA-dependent control of seed germination. The vertical dashed line separates early (left) and late (right) FR-dependent light signaling events. (Left) An early FR pulse inactivates phyB in the endosperm. This leads to PIF1 stabilization in the endosperm and to PIF1-dependent repression of GA3ox1/2 expression (GA) and stimulation of NCED6/9 expression (shown as "ABA" in blue) (Supplemental Fig. 5C). Low GA synthesis prevents testa rupture and stabilizes RGL2, which further stimulates NCED6/9 expression (ABA) (Supplemental Fig. 5A; Piskurewicz et al. 2009). The same early FR pulse activates phyA in the embryo, where a simplified model of the phyA signaling pathway is shown with red lines, emphasizing (1) PIF1 destabilization by the active Pfr form of phyA; (2) PIF1-dependent stimulation of SOM, GAI, and RGA expression; (3) SOM-dependent repression of GA3ox1/2 (GA) expression and stimulation of NCED6/9 (ABA) expression; (4) GA-dependent GAI and RGA destabiliza- 
preventing germination under canopy light will eventually be outweighed by the disadvantages of preventing vegetative growth, which is essential to complete the plant's life cycle. Therefore, the plant must find a fine balance between maintaining a protective growth arrest and not missing an opportunity to initiate vegetative growth.

A possible avenue of inquiry may be provided by considering how phytochrome-dependent responses happen to be compartmentalized. Our observations suggest the unexpected conclusion that control of germination is extraembryonic, occurring in the endosperm, where ABA is produced and released toward the embryo in response to environment cues. This process is energy-consuming and does not appear to require embryonic food stores. Indeed, we showed that isolated endosperm tissue produces and releases ABA over time and that the seed coats retain their capacity to repress embryonic growth in a SCBA (Fig. 3E; Lee et al. 2010). Therefore, it is tempting to speculate that the available food stores in the endosperm determine the seed's energy potential to control germination. Consistent with this hypothesis, Penfield et al. (2006) observed that ABA could prevent lipid mobilization in the embryo but not in the endosperm. Thus, committing the endosperm to control seed germination would allow the plant to prevent embryonic growth while preserving its food reserves for later growth. This possibility is further substantiated by the observation that coatless embryos are able to develop into young seedlings, suggesting that they contain sufficient energy to fuel their growth. However, these speculations must be tempered by the observation that endospermic food stores can contribute to hypocotyl elongation during etiolation (Penfield et al. 2006).

Finally, the results reported here also raise questions concerning the evolution of light-dependent germination control mechanisms in seed plants. It has been proposed that phyA evolved to allow the plant to adapt to a FRenriched terrestrial world resulting from land plant colonization (Mathews 2006). Our results raise the question about the origin of endospermic germination control in the Arabidopsis lineage. Light-dependent control of seed germination is widespread in seed plant species with widely different life styles and seed anatomies. Whether the compartmentalization and phytochrome pathways operate as in Arabidopsis remains to be explored.

\section{Materials and methods}

\section{Plant materials}

All of the Arabidopsis mutants and transgenic lines used in this study were derived from Col ecotype except abi5-4 (WS ecotype) and were harvested on the same day from plants grown side by side. The aba2-1 mutant was kindly provided by Maarten Koornneef (Koornneef et al. 1984), abi5-7 mutant was provided by Eiji Nambara (Nambara et al. 2002), and the abi5-4 mutant isolation was described by Lopez-Molina and Chua (2000). T-DNA insertional mutant seeds of som (SALK_090314), pif1 (SALK_072677), rgl2 (SALK_027654), gai (SAIL_587_C02), rga (SALK_089146), and ga1 (SALK_109115) were obtained from the Nottingham Arabidopsis Stock Centre (NASC). phyA2-11 and
phyB-9 mutant seeds were obtained from NASC. The phyA-211/ phyC-2/phyD-201/phyE-201 (ACDE), phyB-9/phyC-2/phyD-201/ phyE-201 (BCDE), and phyA-211/phyB-9/phyC-2/phyD-201/ phyE-201 (ABCDE) mutants in $\mathrm{ft}-1$ mutant background were described by Strasser et al. (2010) and were kindly provided by Pablo D. Cerdan.

\section{Growth conditions}

Seeds were plated on MS medium (Sigma-Aldrich) containing $0.8 \%(\mathrm{w} / \mathrm{v})$ agar without stratification procedure. Medium was supplemented with $\mathrm{GA}_{4}+7$ (Duchefa Biochemie), ( \pm )-ABA (Sigma-Aldrich), and norflurazon (Supelco, Inc.) according to the germination condition examined. At $4 \mathrm{~h}$ after seed imbibition under white light, the seeds were irradiated in a growth chamber (CLF Plant Climatics, Percival I-30BLLX) with FR (4.5 $\left.\mu \mathrm{mol} \cdot \mathrm{m}^{-2} \cdot \mathrm{sec}^{-1}\right)$ for $5 \mathrm{~min}$ and $\mathrm{R}\left(20 \mu \mathrm{mol} \cdot \mathrm{m}^{-2} \cdot \mathrm{sec}^{-1}\right)$ for $5 \mathrm{~min}$. Seeds were kept in darkness by wrapping plates in several layers of aluminum foil. For the phyA-dependent germination assay, the seeds were irradiated with first FR $\left(4.5 \mu \mathrm{mol} \cdot \mathrm{m}^{-2} \cdot \mathrm{sec}^{-1}\right)$ for $5 \mathrm{~min}$ and further irradiated with second FR $\left(4.5 \mu \mathrm{mol} \cdot \mathrm{m}^{-2} \cdot \mathrm{sec}^{-1}\right)$ for 30 min after $8 \mathrm{~h}$ or $44 \mathrm{~h}$ of dark incubation. Germination rates were scored on the basis of radical emergence. Between 300 and 400 seeds were used to check radical emergence and repeated three times.

\section{Seed dissection and SCBA}

At $4 \mathrm{~h}$ after seed imbibition, the seeds were dissected into embryos and seed coats as described in Lee et al. (2010). In brief, 12 coatless embryos were laid on a layer of 100 seed coats on the surface of MS agar medium and irradiated with FR or R as described above, when both embryos and seed coats were irradiated with the same light. When the coatless embryos and seed coats were irradiated with different light, each embryo and seed coat was irradiated with FR or R separately and combined under a green safety lamp.

\section{RNA extraction and quantitative RT-PCR}

At least 200 seeds, embryos, and seed coats were used for total RNA extraction. All materials were dissected and collected under a green safety light at the time indicated. Total RNA was extracted by using a Spectrum Plant Total RNA kit (Sigma) and quantified spectrophotometrically at $260 \mathrm{~nm}$. Total RNAs were treated with RQ1 RNase-Free DNase (Promega) and reverse-transcribed by using Improm II reverse transcriptase (Promega) and oligo(dT)15 primer (Promega) according to the manufacturer's recommendations. Quantitative RT-PCR was performed by using the ABI $7900 \mathrm{HT}$ fast real-time PCR system (Applied Biosystems) and Power SYBR Green PCR master mix (Applied Biosystems). Relative transcript level was calculated by using the comparative $\Delta \mathrm{Ct}$ method and normalized to the UBQ11 (At4g05050) or PP2A (At1g69960) gene transcript levels. The primers used in this study were designed by Primer Express software for real-time PCR, version 3.0 (Applied Biosystems) and the primer sequences can be found in Supplemental Table 1.

\section{Protein extraction and Western blot analysis}

All materials were dissected and collected under a green safety light at the time indicated. Total proteins were extracted from 20 seeds, embryos, and seed coats with homogenization buffer $(0.0625 \mathrm{M}$ Tris- $\mathrm{HCl}$ at $\mathrm{pH} 6.8,1 \%[\mathrm{w} / \mathrm{v}]$ SDS, $10 \%$ [v/v] glycerin, $0.01 \%[\mathrm{v} / \mathrm{v}] 2$-mercaptoethanol). The total proteins were separated by SDS-PAGE gel and blotted onto immobilon-P transfer membrane (Milipore). ABi5 protein was immunochemically 
detected as described in Piskurewicz et al. (2008), and PHYA and PHYB proteins were detected as described in Shinomura et al. (1994) and Piskurewicz et al. (2008).

\section{ABA measurements}

In Figure 4B and Supplemental Figure 2C, 500 coatless embryos and 500 seed coats were dissected and collected at the time indicated. In Figure 5C, $\sim 50 \mathrm{mg}$ of Arabidopsis seeds was collected at the time indicated. In Figure 3E, 500 wild-type seed coats were dissected from wild-type seeds $24 \mathrm{~h}$ after imbibition in darkness and were cultured in $300 \mu \mathrm{L}$ of MS medium with $0.16 \%$ agar prior to irradiation with either a FR pulse or FR/R pulse followed by incubation of $32 \mathrm{~h}$ in darkness. Thereafter, seed coat material and the culture medium were harvested separately and used to measure absolute ABA levels. All dissections and collections were performed under a green LED light (wavelength, $525 \mathrm{~nm}$ ). The ABA levels were determined as described in Piskurewicz et al. (2009), and all experiments were repeated twice.

\section{ChIP-seq assay and analysis}

ChIP Immunoprecipitation of in vivo fixed chromatin fragments was performed according to Bowler et al. (2004). The abi54 mutant seeds and abi5-4 seeds expressing estradiol-inducible promoter driving HA-ABI5 (abi5-4/ind::HA-ABI5) were plated on MS medium containing $10 \mu \mathrm{M} 17-\beta$ estradiol (Calbiochem) and 3 $\mu \mathrm{M}( \pm)$-ABA. The estradiol concentrations were adjusted to obtain HA-ABI5 protein levels in abi5-4/ind::HA-ABI5 seeds that are similar to those found in ABA-treated wild-type seeds (data not shown). After $24 \mathrm{~h}$ of seed imbibition, seeds were treated with $1 \%$ formaldehyde for 30 min under vacuum. Crosslinking was blocked, and chromatin was isolated following the protocol of Bowler et al. (2004). Chromatin was sheared by sonication into an average size of 100-200 base pairs (bp). HA:: ABI5 was immunoprecipitated using Anti-HA Affinity Matrix (Roche). Beads were rinsed according to the manufacturer's recommendations, and chromatin was eluted and extracted according to Bowler et al. (2004).

ChIP-seq The immunoprecipitated DNA was prepared for sequencing using the Illumina ChIP-seq sample preparation protocol. In brief, 10 ng of ChIP-enriched DNA was end-repaired, and an adenosine overhang was added to the $3^{\prime}$ ends. Paired-end Illumina adapters were ligated to the DNA, and 300-bp fragments were size-selected from a $2 \%$ agarose gel. The eluted products were enriched using 18 cycles of amplification with Illumina PCR primers. Libraries were validated on the Bioanalyzer 2100 (Agilent) and Qubit fluorimeter (Invitrogen). Each sample was run on one lane (12 pM loaded) of the Illumina Hiseq 2000 according to the single-read/40 cycle protocol.

Peak detection All reads for the three conditions (Exp, Input, and Cont) were mapped to the TAIR10 reference genome using bowtie (Langmead et al. 2009). Enrichment analysis between Exp and Input and between Exp and Cont using MACS (Zhang et al. 2008) provided very similar peak detection results ( $82 \%$ identical peaks). Enrichment analysis between Input and Cont provided only 355 nonsignificant peaks, none of which were detected in the Exp versus Input analysis. Because of the similarity between Input and Cont samples, all further analyses were computed on the Exp versus Input data set only.

Motif discovery A list of 3294 peaks with a $P$-value $<10^{-4}$ were selected, and sequences within $200 \mathrm{bp}$ around each peak summit were extracted for motif search. Repeated elements were masked using RepeatMasker (Open 3.0, http://www.repeatmasker.org), and motif discovery was performed using MDmodule from the motifRegressor suite (Conlon et al. 2003). One motif, discovered in 2074 sequences $(63 \%)$, was significantly enriched with a $P$-value $<10^{-5}$ (Wilcoxon test between Exp and Input score distributions). This motif can be represented by the consensus motif $(\mathrm{G} / \mathrm{T})(\mathrm{A} / \mathrm{C})$ CACGTG(T/G)(C/A). The position weight matrix (PWM) of this motif was used to detect motif occurrences in selected genes (Fig. 6B; Supplemental Fig. 5C).

Peak enrichment The significance of each peak located in the promoter of a selected gene was computed by comparing Exp versus Input fold change with the fold changes in 10,000 random regions selected on the same chromosome and with the same width.

\section{Acknowledgments}

We are especially grateful to Pierre Vassalli, Mathias Zeidler, and Christophe Belin for critical reading of the manuscript and helpful suggestions. We thank Enrico Coen for helpful discussions. We are greatly indebted to the following colleagues for their generous gifts: Maarten Koornneef for the aba2-1 mutant; Eiji Nambara for the abi5-7 mutant; Pablo Cerdan for the $f t-1$, ft-1/phyA-211/phyC-2/phyD-201/phyE-201, ft-1/phyB-9/phyC2/phyD-201/phyE-201, and ft-1/phyA-211/phyB-9/phyC-2/phyD201/phyE-201 mutants; and Akira Nagatani for the phyA and phyB antibodies. We thank Mylène Docquier, Patrick Descombes, and members of the Genomics Platform of the National Research Center Frontiers in Genetics for their invaluable help in conducting and interpreting ChIP-seq experiments. This work was supported by grants from the Swiss National Science Foundation and by the State of Geneva.

\section{References}

Bentsink L, Koornneef M. 2008. Seed dormancy and germination. Arabidopsis Book 6: e0119. doi: 10.1199/tab.0119.

Bowler C, Benvenuto G, Laflamme P, Molino D, Probst AV, Tariq M, Paszkowski J. 2004. Chromatin techniques for plant cells. Plant J 39: 776-789.

Conlon EM, Liu XS, Lieb JD, Liu JS. 2003. Integrating regulatory motif discovery and genome-wide expression analysis. Proc Natl Acad Sci 100: 3339-3344.

Dechaine JM, Gardner G, Weinig C. 2009. Phytochromes differentially regulate seed germination responses to light quality and temperature cues during seed maturation. Plant Cell Environ 32: 1297-1309.

Finkelstein R, Reeves W, Ariizumi T, Steber C. 2008. Molecular aspects of seed dormancy. Annu Rev Plant Biol 59: 387-415.

Franklin KA, Praekelt U, Stoddart WM, Billingham OE, Halliday KJ, Whitelam GC. 2003. Phytochromes B, D, and E act redundantly to control multiple physiological responses in Arabidopsis. Plant Physiol 131: 1340-1346.

Hennig L, Stoddart WM, Dieterle M, Whitelam GC, Schafer E. 2002. Phytochrome E controls light-induced germination of Arabidopsis. Plant Physiol 128: 194-200.

Kami C, Lorrain S, Hornitschek P, Fankhauser C. 2010. Lightregulated plant growth and development. Curr Top Dev Biol 91: 29-66.

Kim DH, Yamaguchi S, Lim S, Oh E, Park J, Hanada A, Kamiya Y, Choi G. 2008. SOMNUS, a CCCH-type zinc finger protein in Arabidopsis, negatively regulates light-dependent seed germination downstream of PIL5. Plant Cell 20: 1260-1277. 
Lee et al.

Koornneef M, Reuling G, Karssen CM. 1984. The isolation and characterization of abscisic acid-insensitive mutants of Arabidopsis thaliana. Physiol Plant 61: 377-383.

Langmead B, Trapnell C, Pop M, Salzberg SL. 2009. Ultrafast and memory-efficient alignment of short DNA sequences to the human genome. Genome Biol 10: R25. doi: 10.1186/gb-200910-3-r25.

Lee KP, Piskurewicz U, Tureckova V, Strnad M, Lopez-Molina L. 2010. A seed coat bedding assay shows that RGL2-dependent release of abscisic acid by the endosperm controls embryo growth in Arabidopsis dormant seeds. Proc Natl Acad Sci 107: 19108-19113.

Linkies A, Graeber K, Knight C, Leubner-Metzger G. 2010. The evolution of seeds. New Phytol 186: 817-831.

Lopez-Molina L, Chua NH. 2000. A null mutation in a bZIP factor confers ABA-insensitivity in Arabidopsis thaliana. Plant Cell Physiol 41: 541-547.

Lopez-Molina L, Mongrand S, Chua NH. 2001. A postgermination developmental arrest checkpoint is mediated by abscisic acid and requires the ABI5 transcription factor in Arabidopsis. Proc Natl Acad Sci 98: 4782-4787.

Lopez-Molina L, Mongrand S, McLachlin DT, Chait BT, Chua NH. 2002. ABI5 acts downstream of ABI3 to execute an ABA-dependent growth arrest during germination. Plant J 32: 317-328.

Mathews S. 2006. Phytochrome-mediated development in land plants: Red light sensing evolves to meet the challenges of changing light environments. Mol Ecol 15: 3483-3503.

Muller K, Tintelnot S, Leubner-Metzger G. 2006. Endospermlimited Brassicaceae seed germination: Abscisic acid inhibits embryo-induced endosperm weakening of Lepidium sativum (cress) and endosperm rupture of cress and Arabidopsis thaliana. Plant Cell Physiol 47: 864-877.

Nambara E, Suzuki M, Abrams S, McCarty DR, Kamiya Y, McCourt P. 2002. A screen for genes that function in abscisic acid signaling in Arabidopsis thaliana. Genetics 161: 12471255.

Oh E, Kim J, Park E, Kim J-I, Kang C, Choi G. 2004. PIL5, a phytochrome-interacting basic helix-loop-helix protein, is a key negative regulator of seed germination in Arabidopsis thaliana. Plant Cell 16: 3045-3058.

Oh E, Yamaguchi S, Kamiya Y, Bae G, Chung WI, Choi G. 2006. Light activates the degradation of PIL5 protein to promote seed germination through gibberellin in Arabidopsis. Plant $J$ 47: 124-139.

Oh E, Yamaguchi S, Hu J, Yusuke J, Jung B, Paik I, Lee HS, Sun TP, Kamiya Y, Choi G. 2007. PIL5, a phytochrome-interacting bHLH protein, regulates gibberellin responsiveness by binding directly to the GAI and RGA promoters in Arabidopsis seeds. Plant Cell 19: 1192-1208.

Park J, Lee N, Kim W, Lim S, Choi G. 2011. ABI3 and PIL5 collaboratively activate the expression of SOMNUS by directly binding to its promoter in imbibed Arabidopsis seeds. Plant Cell 23: 1404-1415.

Penfield S, Li Y, Gilday AD, Graham S, Graham IA. 2006. Arabidopsis ABA INSENSITIVE4 regulates lipid mobilization in the embryo and reveals repression of seed germination by the endosperm. Plant Cell 18: 1887-1899.

Piskurewicz U, Jikumaru Y, Kinoshita N, Nambara E, Kamiya Y, Lopez-Molina L. 2008. The gibberellic acid signaling repressor RGL2 inhibits Arabidopsis seed germination by stimulating abscisic acid synthesis and ABI5 activity. Plant Cell 20: 2729-2745.

Piskurewicz U, Tureckova V, Lacombe E, Lopez-Molina L. 2009. Far-red light inhibits germination through DELLA-dependent stimulation of ABA synthesis and ABI3 activity. EMBO J 28: 2259-2271.
Poppe C, Schafer E. 1997. Seed germination of Arabidopsis thaliana phyA/phyB double mutants is under phytochrome control. Plant Physiol 114: 1487-1492.

Reed JW, Nagatani A, Elich TD, Fagan M, Chory J. 1994. Phytochrome A and Phytochrome B have overlapping but distinct functions in Arabidopsis development. Plant Physiol 104: 1139-1149.

Rockwell NC, Su YS, Lagarias JC. 2006. Phytochrome structure and signaling mechanisms. Annu Rev Plant Biol 57: 837-858.

Shinomura T, Nagatani A, Chory J, Furuya M. 1994. The induction of seed germination in Arabidopsis thaliana is regulated principally by Phytochrome B and secondarily by Phytochrome A. Plant Physiol 104: 363-371.

Shinomura T, Nagatani A, Hanzawa H, Kubota M, Watanabe M, Furuya M. 1996. Action spectra for phytochrome A- and Bspecific photoinduction of seed germination in Arabidopsis thaliana. Proc Natl Acad Sci 93: 8129-8133.

Strasser B, Sanchez-Lamas M, Yanovsky MJ, Casal JJ, Cerdan PD. 2010. Arabidopsis thaliana life without phytochromes. Proc Natl Acad Sci 107: 4776-4781.

Weitbrecht K, Muller K, Leubner-Metzger G. 2011. First off the mark: Early seed germination. I Exp Bot 62: 3289-3309.

Yamaguchi S, Smith MW, Brown RG, Kamiya Y, Sun T. 1998. Phytochrome regulation and differential expression of gibberellin $3 \beta$-hydroxylase genes in germinating Arabidopsis seeds. Plant Cell 10: 2115-2126.

Zhang Y, Liu T, Meyer CA, Eeckhoute J, Johnson DS, Bernstein BE, Nusbaum C, Myers RM, Brown M, Li W, et al. 2008. Model-based analysis of ChIP-seq (MACS). Genome Biol 9: R137. doi: 10.1186/gb-2008-9-9-r137. 


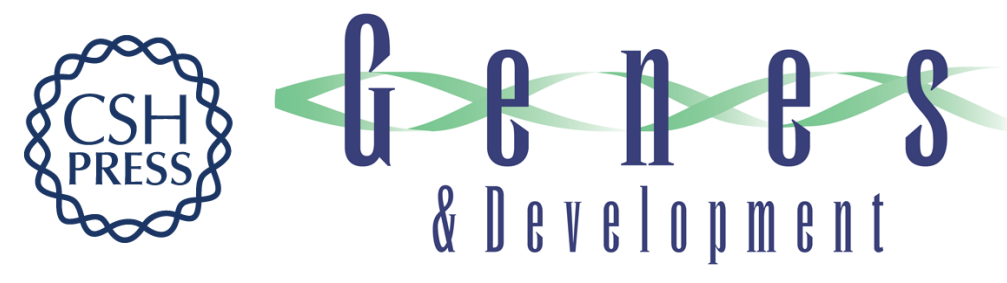

\section{Spatially and genetically distinct control of seed germination by phytochromes A and B}

Keun Pyo Lee, Urszula Piskurewicz, Veronika Turecková, et al.

Genes Dev. 2012, 26:

Access the most recent version at doi:10.1101/gad.194266.112

Supplemental http://genesdev.cshlp.org/content/suppl/2012/08/28/26.17.1984.DC1
Material

References This article cites 35 articles, 17 of which can be accessed free at:

http://genesdev.cshlp.org/content/26/17/1984.full.html\#ref-list-1

License

Email Alerting Receive free email alerts when new articles cite this article - sign up in the box at the top

Service right corner of the article or click here.

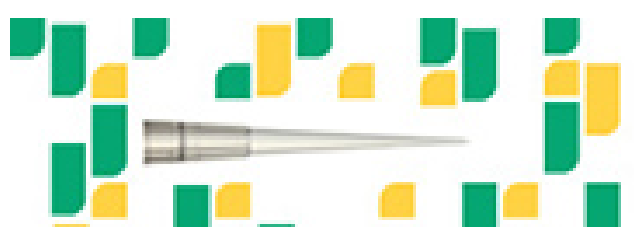

Focused on your science. 